\title{
Preparation of Eucommia ulmoides Leaves Antioxidant and Its Antioxidation Activity In Vitro and In Vivo
}

\author{
Wenbin Dong ${ }^{1,2}$, Di Liu ${ }^{*}, 2$, Jin Yang ${ }^{1}$ and Xianmeng $\mathrm{Xu}^{1}$ \\ ${ }^{I}$ College of Life Science and Engineering, Shaanxi University of Science and Technology, Xi'an, Shaanxi, 710021, P.R \\ of China \\ ${ }^{2}$ College of Chemistry and Chemical Industry, Shaanxi University of Science and Technology, Xi'an, Shaanxi, 710021, \\ P.R. of China
}

\begin{abstract}
The adsorption and desorption efficiencies on flavonoids and polyphenols from Eucommia ulmoides leaves by different resins were investigated, and the most suitable resin for preparation of Eucommia ulmoides leaves antioxidant was chosen. Then the crude extract of Eucommia ulmoides leaves was adsorbed by resin, and the resin was eluted by $20 \%$, $40 \%, 60 \%$ and $80 \%$ (in volume) ethanol elution solution, and then the antioxidants were gotten. The flavonoids and polyphenols in the antioxidants were tested for content. The antioxidation activity in vitro was studied by testing the scavenging capacities on $\mathrm{HO}, \mathrm{O}_{2}{ }^{-}$and $\mathrm{DPPH} \cdot$. The antioxidation activity in vivo was studied by measuring the content of malondialchehyche (MDA), and superoxide dismutase (SOD) and glutathione peroxidase (GSH-PX) activity in mice orally administrated by $20 \%$ and $60 \%$ ethanol elution solution sample. The result showed: the $20 \%, 40 \%$ and $60 \%$ ethanol elution solution had good scavenging capacities on $\mathrm{HO} \cdot \mathrm{O}_{2}{ }^{-} \cdot$ and $\mathrm{DPPH} \cdot$, and the antioxidation activities in vitro of the antioxidants was related to flavonoids and polyphenols, and polyphenols was the leading working material in antioxidation in vitro; $20 \%$ ethanol elution solution, of which there was more polyphenols in, showed more obvious antioxidation activities in vivo, which also meant that polyphenols was the leading working material in antioxidation in vivo.
\end{abstract}

Keywords: Eucommia ulmoides leaves, resin, antioxidant, antioxidation activity.

\section{INTRODUCTION}

Eucommia ulmoides is an endemic and economic useful tree in China, of which the extract has complex components and multiplex function [1-8]. In China, the bark of Eucommia ulmoides tree has been used as herb for two thousands years. The recent researches show that the leaves of Eucommia ulmoides tree have the similar components to bark, and the function is also similar, so leaves can be also used as herb instead of bark [9]. This provides a wide space for the comprehensive using of Eucommia ulmoides. There are more flavonoids and polyphenols in Eucommia ulmoides leaves [9], which can exhibit obvious antioxidation activity. Some researches show that Eucommia ulmoides has stronger capacity on scavenging free radicals [10], but the most researches objects are crude extract. In this study, we used resin to separate and purify Eucommia ulmoides leaves crude extract to prepare antioxidant, which was first researched as object to study Eucommia ulmoides leaves antioxidation activities in vitro and in vivo. At last, the antioxidation activities in vitro and in vivo of different antioxidants from Eucommia ulmoides leaves were evaluated.

*Address correspondence to this author at the College of Life Science and Engineering, Shaanxi University of Science and Techonolgy, Xi'an, Shaanxi, 710021, P.R of China; Tel: 0086 15929293600; Fax: 008629 3357280; E-mail: didi19801210@163.com

\section{MATERIALS AND METHODS}

\subsection{Chemicals}

LS-46 resin was supplied by Xi' an Lanshen Science and Technology Co., Ltd; HPD-600 resin was supplied by Hebei Cangzhou Baoen Chemical Co. Ltd; XDA-8 resin was supplied by Xi' an Lanxiao Science and Technology Co. Ltd. 1,1-Diphenyl-2-picrylhydrazyl (DPPH) was provided by Sigma-Aldric Company. All the kits were purchased from Nanjing Jiancheng Bioengineering Institute, Nanjing, China.

\subsection{Animal}

Male Clean Kunming mice weighing $20 \pm 2.0 \mathrm{~g}$ (Experimental Animal Centre, Medical College of Xi'an Jiaotong University) were used in these experiments.

\subsection{Preparation for Eucommia Ulmoides Leaves Crude Extract}

After Eucommia ulmoides leaves were washed, they were dried at $50^{\circ} \mathrm{C}$, and then crushed into powder. The powder was mixed with extraction solvent in 1:10, and the mixture was heated at $50^{\circ} \mathrm{C}$ in water bath for $1 \mathrm{~h}$. The extraction solution was filtered and the filtrate was collected and centrifuged. The supernatant liquor was crude extract liquor. The crude extract liquor was concentrated and dried in vacuum to become powder sample. 


\subsection{Choosing Resin}

LS-46、HPD-600and XDA-8 resins were prepared for testing. Static adsorption method was used to test adsorption efficiency of each type resin on flavonoids and ployphenols, and dynamic desorption method was used to test desorption efficiency of each type resin on flavonoids and ployphenols. Then based on adsorption and desorption efficiency, the suitable resin was chosen for separation and purification of Eucommia ulmoides leaves antioxidant.

\subsection{Preparation of Eucommia ulmoides Leaves Antioxidant}

The prepared resin was put into column, and $1 \mathrm{BV}$ crude extract liquor flowed through the resin in $1 \mathrm{BV} / \mathrm{h}$ flow rate for adsorption, and each 4BV $20 \%, 40 \%, 60 \%$ and $80 \%$ (in volume) ethanol elution solution flowed through the resin in sequence with $1 \mathrm{BV} / \mathrm{h}$ flow rate for desorption. Each ethanol elution solution flowing through the resin was collected, and then they were concentrated and dried in vacuum to become antioxidant samples in powder.

\subsection{Flavonoids and Polyphenols Measurement}

Each sample was measured for flavonoids with $\mathrm{Al}\left(\mathrm{NO}_{3}\right)_{3}$ method [11] using rutin as standard and ployphenols with Folin-Ciocalteu method [12] using gallic acid as standard.

\subsection{Antioxidation Activity In vitro}

Each sample, including crude extract and antioxidant samples, was prepared to $0.1 \mathrm{mg} / \mathrm{mL}$ water solution. The scavenging efficiencies on different free radicals were measured, and $\mathrm{Vc}$ was taken as a reference sample. The scavenging capacities on $\mathrm{HO} \cdot \mathrm{O}_{2}^{-} \cdot$ and $\mathrm{DPPH} \cdot$ were measured respectively as references [13] stated.

\subsection{Animal Grouping and Administration}

Mice weighed before experiment, were randomly divided into 3 groups at random (30 mice/group), including $\mathrm{NC}$ (negative control) group, EUI (20\% ethanol elution) group and EUII (60\% ethanol elution) group. All the mice were fed in laboratory animal room, where the temperature was kept in $25.0 \pm 2.0^{\circ} \mathrm{C}$ and relative humidity was kept in $40-60 \%$. The mice were kept in separated cage and fed with standard feed. The mice freely drank water and ate feed. Before oral administration test, the mice were adapted in laboratory animal room for $3 \mathrm{~d}$. During the oral administration test, the mice were taken swimming exercise for $30 \mathrm{~min}$ in the same time everyday. EUI and EUII were dissolved with pure water to $0.2 \mathrm{~g} / \mathrm{ml}$ solution. The oral administration dose is 0.01 $\mathrm{ml} / 10 \mathrm{~g}$ everyday. The extracts were orally administered into mice in EUI and EUII group once per day for $15 \mathrm{~d}$ using a feeding atraumatic needle.

\subsection{Measurement of MDA, SOD, GSH-PX [14-16]}

1 hour after the last oral administration, the mice (10 mice picked at random from per group) were forced to swim for $90 \mathrm{~min}$ without weight loading. The mice were anesthetized with ether and whole blood samples were collected in the tubes by the aorta puncture. The blood samples were anti-coagulated with heparin sodium in the tubes. Blood samples were centrifuged for $10 \mathrm{~min}$ at a speed of $4000 \mathrm{rpm}$. The serum was collected and the contents of MDA, the activity of SOD and GSH-PX were analyzed by spectrophotometric method with commercial kit.

\subsection{Statistical Analysis}

The data were expressed as mean \pm standard error of the mean (mean \pm S.D.). Data were analyzed using Student's $t$ test. Differences at $p<0.05$ were considered to be significant.

\section{RESULTS AND DISCUSSION}

\subsection{Adsorption Efficiency of Each Resin}

The adsorption efficiencies of LS-46、HPD-600 and XDA-8 resin on flavonoids and ployphenols from Eucommia ulmoides leaves crude extract were shown in Table 1. Table 1 showed that LS-46 had higher adsorption efficiency on flavonoids, and XDA-8 resin had higher adsorption efficiency on ployphenols

Table 1. Adsorption Efficiency of Resin on Flavonoids and Polyphenols

\begin{tabular}{|c|c|c|}
\hline \multirow{2}{*}{ Resin } & \multicolumn{2}{|c|}{ Adsorption Efficiency (\%) } \\
\cline { 2 - 3 } & Flavonoids & Ployphenols \\
\hline \hline LS-46 & 82.66 & 64.63 \\
HPD-600 & 63.5 & 58.13 \\
XDA-8 & 81.2 & 68.75 \\
\hline
\end{tabular}

\subsection{Adsorption Efficiency of Each Resin}

The desorption efficiency of LS-46、HPD-600 and XDA-8 resin on flavonoids and ployphenols from Eucommia ulmoides leaves crude extract was shown in Table 2. XDA-8 resin had higher desorption efficiency on flavonoids and ployphenols. Following the results showed in Tables $\mathbf{1}$ and $\mathbf{2}$, XDA-8 was chosen for preparation of Eucommia ulmoides leaves antioxidant.

Table 2. Desorption Efficiency of Resin on Flavonoids and Polyphenols

\begin{tabular}{|c|c|c|c|c|c|c|}
\hline \multirow{2}{*}{ Ethanol/\% } & \multicolumn{2}{|c|}{$\begin{array}{c}\text { HS-46 } \\
\text { HPD-600 }\end{array}$} & \multicolumn{2}{c|}{$\begin{array}{c}\text { XDA-8 } \\
\text { Desorption Efficiency (\%) }\end{array}$} \\
\cline { 2 - 7 } & Desorption Efficiency (\%) & Desorption Efficiency (\%) & Polyphenols & Flavonoids & Polyphenols \\
\hline \hline 20 & 6.95 & 23.59 & 11.56 & 20.99 & 11.78 & 44.38 \\
40 & 14.59 & 15.36 & 22.43 & 15.53 & 26.80 & 13.01 \\
60 & 21.42 & 15.21 & 12.65 & 15.21 & 46.86 & 19.07 \\
80 & 5.31 & 9.90 & 1.72 & 6.32 & 2.43 & 4.98 \\
\hline
\end{tabular}




\subsection{Flavonoids and Ployphenols Contents in Different Samples}

Flavonoids and Ployphenols Contents in Different Samples were shown in Fig. (1).

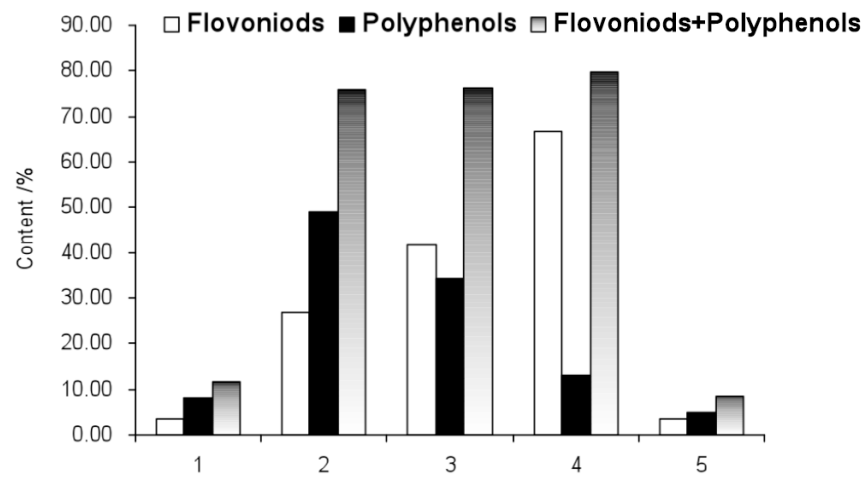

Fig. (1). Flavonoids and polyphenols contents in different samples. 1, crude extract; 2, 20\% ethanol elution solution; 3, 40\% ethanol elution solution; 4, 60\% ethanol elution solution; 5, $80 \%$ ethanol elution solution.

It was shown in Fig. (1) that $20-60 \%$ ethanol elution solution enriched much more flavonoids and polyphenols, and the sums of contents of flavonoids and polyphenols in $20-60 \%$ ethanol elution solution were similar. However, the contents of flavonoids and polyphenols were changed with ethanol content. Polyphenols changed from high content to low content in $20 \%, 40 \%$ and $60 \%$ ethanol elution solution; Flavonoids changed from high content to low content in $60 \%, 40 \%$ and $20 \%$ ethanol elution solution. The result showed that polyphenols were separated first by low content ethanol elution solution (20\%), but flavonoids were separated secondly by high content ethanol elution solution $(60 \%)$. Therefore, when resin was used in separation and purification of flavonoids and polyphenols, each ethanol elution solution could be collected respectively to get products of different component content. In $80 \%$ ethanol elution solution, no matter flavonoids or polyphenols, the content was lowest and near to the content in crude extract.

\subsection{The Antioxidation In Vitro of Each Antioxidant}

The scavenging efficiencies on $\cdot \mathrm{OH}$ from different samples were shown in Fig. (2). Compared with crude extract, the scavenging efficiencies on $\mathrm{OH}$ from $20 \%$ ethanol elution solution sample was increased much significantly $(p<0.01)$, and the scavenging efficiencies on . OH from $40 \%$ and $60 \%$ ethanol elution solution sample was increased significantly $(p<0.05)$, but for $80 \%$ ethanol elution solution sample and $\mathrm{Vc}$, the differences were not obvious $(p>0.05)$; compared with $\mathrm{Vc}$, the scavenging efficiencies on -OH from $20 \%$ ethanol elution solution sample was much significantly higher $(p<0.01)$, and the scavenging efficiencies on $\mathrm{OH}$ from $40 \%$ and $60 \%$ ethanol elution solution sample were significantly higher $(p<0.05)$, but for $80 \%$ ethanol elution solution sample, the difference was not obvious $(p>0.05)$. This showed that after the Eucommia ulmoides leaves crude extract was separated and purified by resin, the antioxidative components were concentrated, and the fact that the scavenging efficiencies on $\cdot \mathrm{OH}$ from $20 \%, 40 \%$ and $60 \%$ ethanol elution solution sample were higher than crude extract proved this conclusion. $\mathrm{Vc}$ is a kind of strong and natural antioxidant. Fig. (2) showed that the scavenging capacities on $\mathrm{OH}$ from $20 \%, 40 \%$ and $60 \%$ ethanol elution solution sample were higher than Vc, especially for $20 \%$ ethanol elution solution sample, the scavenging capacities was much stronger $\mathrm{Vc}$.

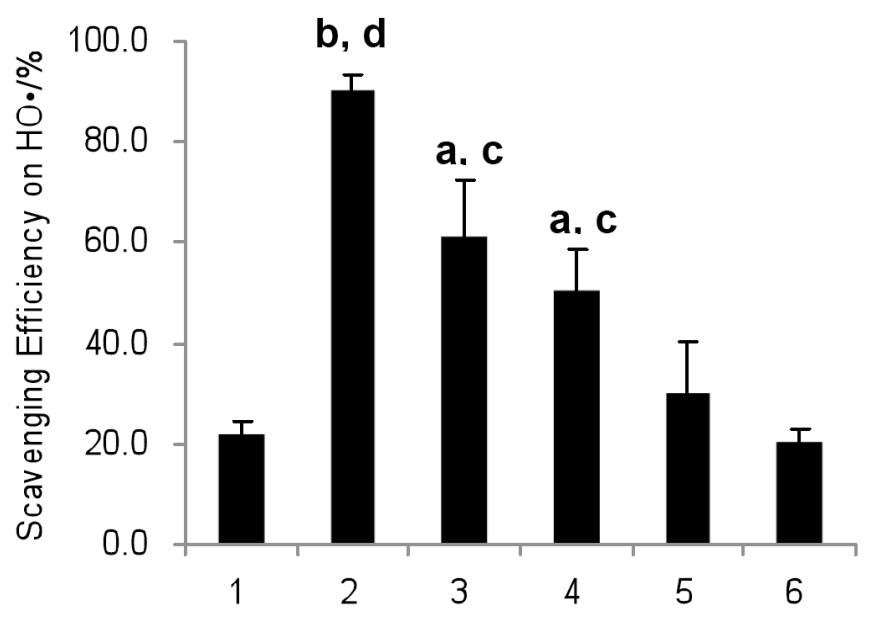

Fig. (2). Scavenging efficiency on $\bullet \mathbf{O H}$ from different samples. 1, crude extract; 2, 20\% ethanol elution solution; 3, 40\% ethanol elution solution; 4, 60\% ethanol elution solution; 5, 80\% ethanol elution solution; 6, Vc. The data were expressed as mean \pm S.D. $(n=3)$. The data were analyzed by t-test. Compared with crude extract, a means $p<0.05$, b means $p<0.01$; compared with $\mathrm{Vc}$, c means $p<0.05$, d means $p<0.01$.

The scavenging efficiencies on $\mathrm{O}_{2}{ }^{-} \cdot$ from different samples were shown in Fig. (3). Compared with crude extract, the scavenging efficiencies on $\mathrm{O}_{2}^{-} \cdot$ from $20 \%$ ethanol elution solution sample was increased much significantly $(p<0.01)$, and the scavenging efficiencies on $\mathrm{O}_{2}^{-}$. from $40 \%$ and $60 \%$ ethanol elution solution sample were increased significantly $(p<0.05)$, but for $80 \%$ ethanol elution solution sample, the difference was not obvious $(p>0.05)$ and for $\mathrm{Vc}$, the difference was much significantly $(p<0.01)$; compared with $\mathrm{Vc}$, the difference of scavenging efficiencies on $\mathrm{O}_{2}{ }^{-}$. between $20 \%$ ethanol elution solution sample and $\mathrm{Vc}$ was not obvious $(p>0.05)$, but for $40 \%, 60 \%$ and $80 \%$ ethanol elution solution sample, the differences were much significant $(p<0.01)$. So, after the Eucommia ulmoides leaves crude extract was separated and purified by resin, except for $80 \%$ ethanol elution solution sample, the scavenging efficiencies on $\mathrm{O}_{2}^{-}$.from each sample were enhanced obviously, especially for $20 \%$ ethanol elution solution sample, its scavenging capacities was much stronger than other samples. However, compared with Vc, which is a strong antioxidant, only $20 \%$ ethanol elution solution sample's scavenging capacity was near to it, and other samples were much weaker than it.

The scavenging efficiencies on DPPH - from different samples were shown in Fig. (4). Compared with crude extract, the scavenging efficiency on DPPH from $20 \%$ ethanol elution solution sample was increased much significantly $(p<0.01)$, and the scavenging efficiencies on $\mathrm{DPPH}$ - from $40 \%$ ethanol elution solution sample was increased significantly $(p<0.05)$ and the difference between $60 \%$ solution sample and crude extract was not obvious, but 


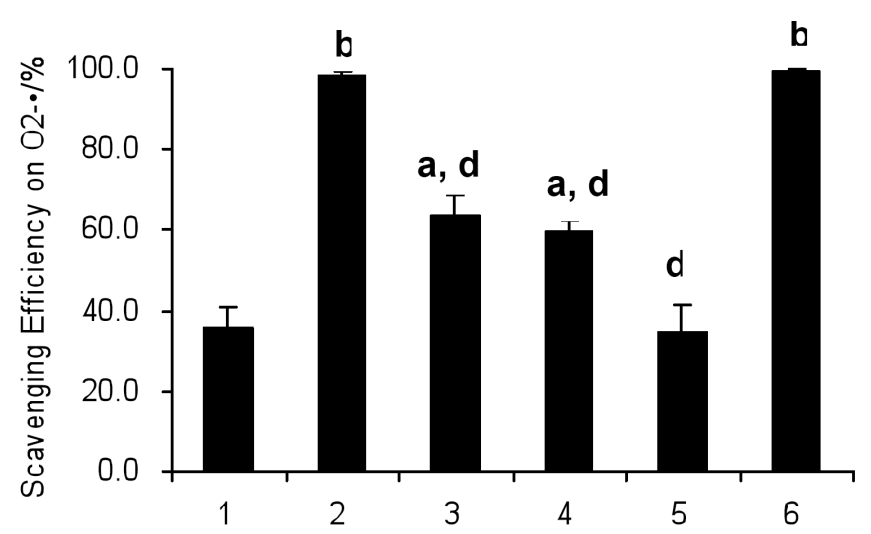

Fig. (3). Scavenging efficiency on $\mathrm{O}_{2}^{-} \cdot$ from different samples. 1 , crude extract; 2, 20\% ethanol elution solution; 3, 40\% ethanol elution solution; 4, 60\% ethanol elution solution; 5, 80\% ethanol elution solution; 6, Vc. The data were expressed as mean \pm S.D. $(n=3)$. The data were analyzed by t-test. Compared with crude extract, a means $p<0.05$, b means $p<0.01$; compared with $\mathrm{Vc}$, c means $p<0.05$, d means $p<0.01$.

for $80 \%$ ethanol elution solution sample, the scavenging efficiency was reduced much significantly $(p<0.01)$ and for $\mathrm{Vc}$, the difference was much significantly $(p<0.01)$; compared with $\mathrm{Vc}$, the difference of scavenging efficiencies on $\mathrm{DPPH} \cdot$ between $20 \%$ ethanol elution solution sample and Vc was not obvious $(p>0.05)$, but for $40 \%, 60 \%$ and $80 \%$ ethanol elution solution sample, the differences were much significant $(p<0.01)$. DPPH is a stable free radical in organic solvent. Except for $80 \%$ ethanol elution solution sample, the scavenging efficiencies on DPPH from each sample were enhanced obviously, especially for $20 \%$ ethanol elution solution sample, its scavenging capacity was much stronger than other samples and near to $\mathrm{Vc}$.

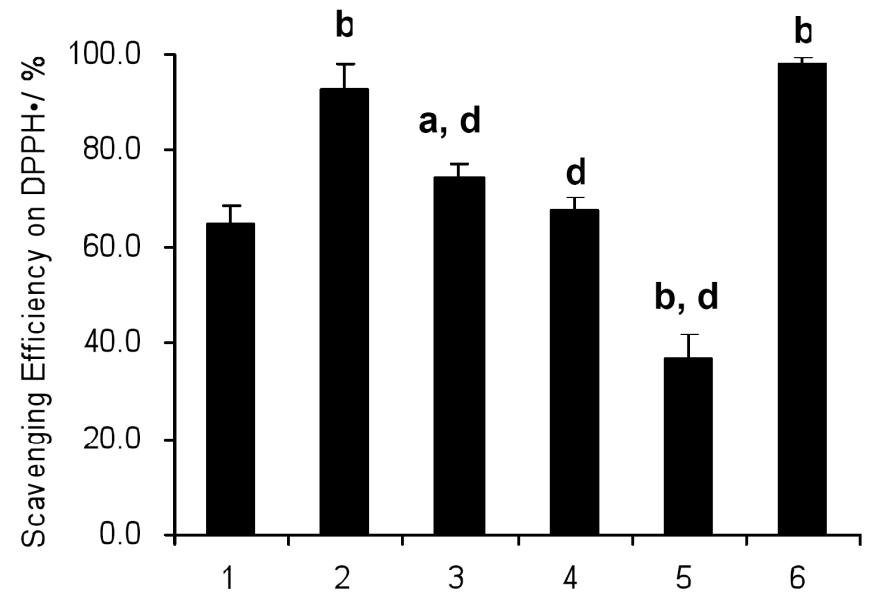

Fig. (4). Scavenging efficiency on DPPH· from different samples. 1 , crude extract; $2,20 \%$ ethanol elution solution; $3,40 \%$ ethanol elution solution; 4, 60\% ethanol elution solution; 5, 80\% ethanol elution solution; 6, Vc. The data were expressed as mean \pm S.D. $(n=3)$. The data were analyzed by t-test. Compared with crude extract, a means $p<0.05$, b means $p<0.01$; compared with $\mathrm{Vc}$, c means $p<0.05$, d means $p<0.01$.

From Figs. (2-4), it could be seen that $20 \%, 40 \%$ and $60 \%$ ethanol elution solution had strong scavenging capacities on each free radicals, but for $80 \%$ ethanol elution solution sample, the difference of scavenging capacity between it and crude extract was not obvious, which was identical to that the contents of flavonoids and polyphenols in $20 \%, 40 \%$ and $60 \%$ ethanol elution solution sample were much higher than $80 \%$ ethanol elution solution sample and crude extract, but for $80 \%$ ethanol elution solution sample, the content was near to crude extract. So, flavonoids and polyphenols were antioxidative component in Eucommia ulmoides leaves. In addition, the sequence of free radicals scavenging capacities from high to low was $20 \%, 40 \%, 60 \%$ and $80 \%$ ethanol elution solution sample, and polyphenols content from high to low was also this sequence. Thus it could be seen that polyphenols played a leading role in antioxidation activity of Eucommia ulmoides leaves antioxidant.

\subsection{The Antioxidation In Vivo of EUI and EUII}

The result of effects on MDA, SOD, and GSH-PX of EUI and EUII in mice was shown in Figs. (5-7). Compared with $\mathrm{NC}$ group, the contents of MDA in blood serum of exercised mice in EUI and EUII group were reduced, and the difference between EUI and NC group was significant $(p<0.05)$, but EUII and NC had no significant difference; the SOD activity of exercised mice in EUI and EUII group were increased, and the difference between EUI and NC group was very significant $(p<0.01)$, but EUII and NC had no significant difference; the GSH-PX activity in both group were increased, and the difference between EUI and NC group was significant $(p<0.05)$, EUII and $\mathrm{NC}$ had no significant difference. In body, SOD can deoxidize $\mathrm{O}^{2-}$ to $\mathrm{H}_{2} \mathrm{O}_{2}$ and $\mathrm{O}_{2}$, and GSH-PX can deoxidize organic peroxide and $\mathrm{H}_{2} \mathrm{O}_{2}$ to $\mathrm{H}_{2} \mathrm{O}$ and non-noxious substance. If there exists SOD and GSH-PX, the content of $\mathrm{O}^{2-}$, organic peroxide and $\mathrm{H}_{2} \mathrm{O}_{2}$ will be reduced. So the peroxidative injury to cell and mitochondrial will be prevented, and MDA, which is the product of lipid perioxidation, will be reduced too. EUI could enhance body's SOD activity $(p<0.01)$, reduced the content of MDA $(p<0.05)$ and increase GSH-PX activity $(p<0.05)$. The result showed that EUI could enhance the antioxidation ability in body.

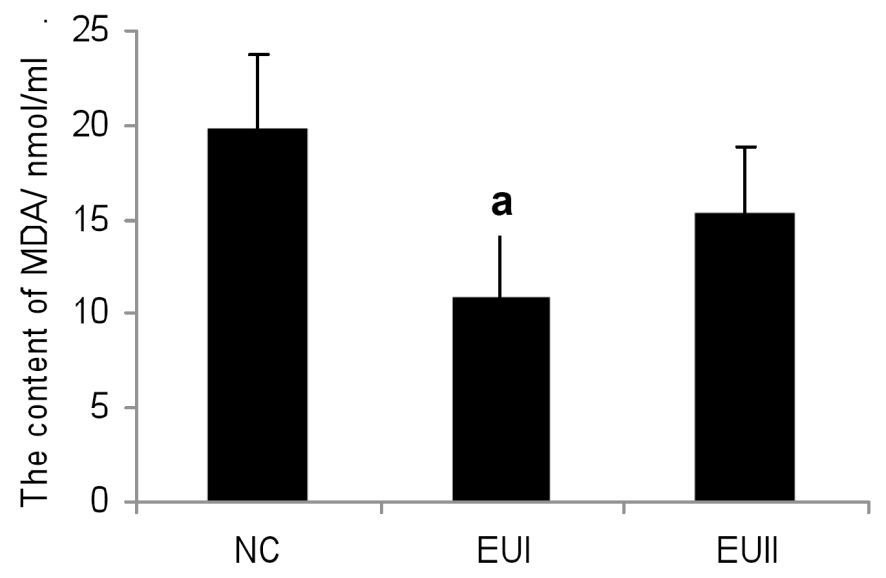

Fig. (5). MDA content in mice. NC, negative group; EUI, 20\% ethanol elution solution group; EUII, $60 \%$ ethanol elution solution group. The data were expressed as mean \pm S.D. $(n=10)$. The data were analyzed by t-test. Compared with $\mathrm{NC}$, a means $p<0.05$, b means $p<0.01$. 


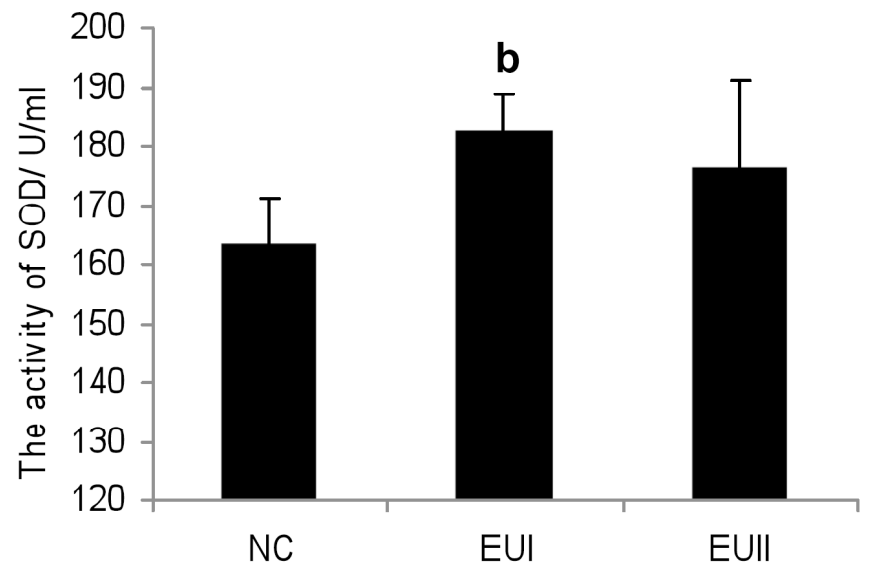

Fig. (6). SOD activity in mice. NC, negative group; EUI, $20 \%$ ethanol elution solution group; EUII, $60 \%$ ethanol elution solution group. The data were expressed as mean \pm S.D. $(n=10)$. The data were analyzed by t-test. Compared with $\mathrm{NC}$, a means $p<0.05, \mathrm{~b}$ means $p<0.01$.

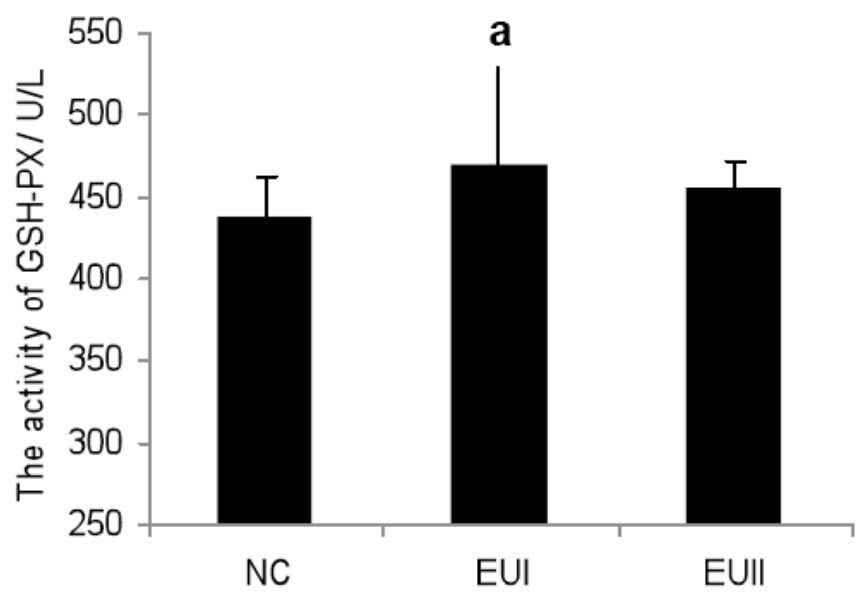

Fig. (7). GSH-PX activity in mice. NC, negative group; EUI, $20 \%$ ethanol elution solution group; EUII, $60 \%$ ethanol elution solution group. The data were expressed as mean \pm S.D. $(n=10)$. The data were analyzed by t-test. Compared with $\mathrm{NC}$, a means $p<0.05, \mathrm{~b}$ means $p<0.01$.

\section{CONCLUSIONS}

- $\quad$ Through the investigation on adsorption efficiency and desorption efficiency on flavonoids and polyphenols from Eucommia ulmoides leaves crude extract by LS-46、HPD-600 and XDA-8 resin, the XDA-8 resin was chosen as a suitable resin for preparation of Eucommia ulmoides leaves antioxidant.

- $\quad$ Except $80 \%$ ethanol elution sample, each antioxidant had much more flavonoids and polyphenols, which were active component on scavenging free radicals, and polyphenols was the leading component. Different ethanol elution solution had different content of flavonoids and polyphenols. In $20 \%$ ethanol elution solution sample, polyphenols were enriched more; in $60 \%$ ethanol elution solution sample, flavonoids were enriched more.
- $\quad$ For antioxidation in vitro, the $20 \%, 40 \%, 60 \%$ and $80 \%$ ethanol elution solution sample had different scavenging capacities on $\mathrm{HO} \cdot \mathrm{O}_{2}^{-} \cdot$ and $\mathrm{DPPH} \cdot$, and $20 \%, 40 \%$ and $60 \%$ ethanol elution solution sample exhibited obviously better scavenging capacities than crude extract. However, $20 \%$ ethanol elution solution sample showed more obvious antioxidation in vitro than others, which showed polyphenols was the leading working material in antioxidation in vitro.

- $\quad$ For antioxidation in vivo, EUI (20\% ethanol elution solution) showed more obvious antioxidation activities in vivo, which also meant that polyphenols was the leading working material in antioxidation in vivo.

\section{ACKNOWLEDGEMENT}

We would like to express our great thanks to the financial support from Science and Technology Innovation Fund of Shaanxi University of Science and Technology.

\section{REFERENCES}

[1] Cheng GL. The analysis of active ingredients from Eucommia ulmoides and its pharmacological research progress. Chin Tradit Pat Med 2006; 28: 723-25.

[2] Luo LF, Wu WH, Zhou YJ, Yan J, Yang GP, Ouyang DS Antihypertensive effect of Eucommia ulmoides Oliv. extracts in spontaneously hypertensive rats. J Ethnopharmacol 2010; 129: 23843.

[3] Kwan CY, Chen CX, Deyama T, Nishibe S. Endotheliumdependent vasorelaxant effects of the aqueous extracts of the Eucommia ulmoides Oliv. leaf and bark: implications on their antihypertensive action. Vasc Pharmacol 2003; 40: 229-35.

[4] Kim HY, Moon BH, Lee HJ, Choi DH. Flavonol glycosides from the leaves of Eucommia ulmoides $O$. with glycation inhibitory activity. J Ethnopharmacol 2004; 93: 227-30.

[5] Lee MK, Kim MJ, Cho SY, et al. Hypoglycemic effect of Duzhong (Eucommia ulmoides Oliv.) leaves in streptozotocin-induced diabetic rats. Diabetes Res Clin Pract 2005; 67: 22-8.

[6] Jin X, Amitani K, Zamami Y, et al. Ameliorative effect of Eucommia ulmoides Oliv. leaves extract (ELE) on insulin resistance and abnormal perivascular innervation in fructosedrinking rats. J Ethnopharmacol 2010; 128: 672-8.

[7] Hung MY, Fu TYC, Shih PH, Lee CP, Yen GC. Du-Zhong (Eucommia ulmoides Oliv.) leaves inhibits CCl4-induced hepatic damage in rats. Food Chem Toxicol 2006; 44: 1424-31.

[8] Zhang R, Liu ZG, Li C, et al. Du-Zhong (Eucommia ulmoides Oliv.) cortex extract prevent OVX-induced osteoporosis in rats. Bone 2009; 45: 553-9.

[9] Zhang J., Gao Z., Zhang Q., Jang Y., Niu A., Hu Y. Study on nutritional and medical components of Eucommia ulmoides leaves and their extracts. Amino Acids Biotic Resour 2006; 24: 1-2.

[10] Kulomaa A, Siren H, Riekkola ML. Identification of antioxidative compounds in plant beverages by capillary electrophoresis with the marker index technique. J Chromatogr A 1997; 781: 523-32.

[11] Fu GM, Wan Y, Zhou JB. Comparison of methods of determining the content of flavonoids in extracts from Evcommia vlmoides oliv. leaves. J Chin Inst Food Sci Technol 2006; 1: 224-9.

[12] Liu LX, Laura T, Liang XF, Sun Y, Sun H, Zeng XX. Determination of the total content of polyphenols in Kudincha by Folin-Ciocalteu colorimetry. J Tea Sci 2008; 2: 101-6.

[13] Zhi J, Li BQ, Lian YJ, Hong L, Xia XF, Tong DJ. Study on polysaccharides extraction from Eleagnus angustifolius L.and their antioxidation activity. Chin Tradit Pat Med 2009; 31: 796-8.

[14] Wang J, Li SS, Fan YY, et al. Anti-exercise fatigue activity of the water-soluble polysaccharides isolated from Panax ginseng C. A. Meyer. J Ethnopharmacol 2010; 130: 421-3. 
[15] Yang CX, Zhai WJ. Study 011 Anti-exercise fatigue Effect of Extract from Eucommia ulmoides Oliver Leaves. Food Sci 2008; 29: $550-2$.
[16] You LJ, Zhao MM, Regenstein JM, Ren JY. In vitro antioxidant activity and in vivo anti-exercise fatigue effect of loach (Misgurnus anguillicaudatus) peptides prepared by papain digestion. Food Chem 2011; 124: 188-94

(C) Dong et al.; Licensee Bentham Open.

This is an open access article licensed under the terms of the Creative Commons Attribution Non-Commercial License (http://creativecommons.org/licenses/ by$\mathrm{nc} / 3.0 /$ ) which permits unrestricted, non-commercial use, distribution and reproduction in any medium, provided the work is properly cited. 